Motoko Tsuruta • Hiroshi Mitsubuchi • Sek Mardy

Yuichi Miura • Yumi Hayashida • Akihiko Kinugasa

Takateru Ishitsu • Ichiro Matsuda • Yasuhiro Indo

\title{
Molecular basis of intermittent maple syrup urine disease: novel mutations in the $E 2$ gene of the branched-chain $\alpha$-keto acid dehydrogenase complex
}

Received: October 29, 1997 / Accepted: November 27, 1997

\begin{abstract}
The E2 gene of the branched-chain $\alpha$-keto acid dehydrogenase (BCKDH) complex was studied at the molecular level in three patients with intermittent maple syrup urine disease (MSUD). All three patients had higher BCKDH activity than did those with the classical phenotype. In the first patient, a single base substitution from A to $\mathrm{G}$ in intron 8 created a new $5^{\prime}$ splice site and caused an insertion of 126 nucleotides between exons 8 and 9 by activating an upstream cryptic $3^{\prime}$ splice site in the same intron. The predicted mRNA encoded a truncated protein with 282 amino acids including 4 novel ones at the carboxyl terminus, compared with the normal protein with 421 amino acids. In vitro, the region from the patient but not from a normal control was recognized and was recovered as a novel exon, indicating that the single substitution was responsible for incorporation of the region into mRNA. This mutation probably supports an exon definition model in which the spliceosome recognizes a $3^{\prime}$ splice site and then scans downstream for an acceptable $5^{\prime}$ splice site, thereby defining an exon. The second patient was homozygous for a $G$ to $T$ transversion at nucleotide 1463 in exon 11, which predicted a substitution of the termination codon by a leucine residue and the addition of 7 extra amino acids at the carboxyl terminus. For each mutation, these two patients were homozygous and their parents were heterozygous. The third patient was a compound heterozygote for a $\mathrm{C}$ to $\mathrm{G}$ transversion at nucleotide 309 in exon 4 and a $G$ to A transition at nucleotide 1165 in exon 9 , causing an Ile-toMet substitution at amino acid 37 and a Gly-to-Ser substitu-
\end{abstract}

M. Tsuruta $\cdot$ H. Mitsubuchi $\cdot$ S. Mardy $\cdot$ Y. Miura $\cdot$ Y. Hayashida $\cdot$

I. Matsuda $\cdot$ Y. Indo $(\square)$

Department of Pediatrics, Kumamoto University School of

Medicine, Honjo 1-1-1, Kumamoto 860-8556, Japan

Tel. +81-96-373-5191; Fax +81-96-366-3471

A. Kinugasa

Department of Pediatrics, Kyoto Prefectural University of Medicine,

Kyoto, Japan

T. Ishitsu

Department of Pediatrics, National Saishunso Hospital, Kumamoto, Japan tion at amino acid 323 , respectively. Taken together, these results indicate that the molecular basis of intermittent phenotype MSUD in some patients can be due to mutations in the E2 gene, giving rise to a low but significant residual activity of the BCKDH complex.

Key words Maple syrup urine disease - Intermittent maple syrup urine disease - Branched-chain $\alpha$-keto acid dehydrogenase complex $\cdot$ Dihydrolipoyl transacylase (E2) - Inborn error of metabolism - Mutation analysis of the E2 gene

\section{Introduction}

Maple syrup urine disease (MSUD) is an autosomal recessive inborn error of metabolism characterized by ketoacidosis, distinctive odor of the urine, and in some cases by developmental retardation. Several phenotypes of MSUD have been distinguished on the basis of clinical features: classical, intermittent, intermediate, and thiamineresponsive types (Chuang and Shih 1995). This classification is based on the rapidity of onset, severity of the disease, tolerance for dietary protein, response to thiamine supplements, and the results of enzyme assays, which usually show higher activity in the intermittent than in the classical phenotype. Patients with the intermittent phenotype of MSUD are unique, since their laboratory data, including blood branched-chain amino acid levels, are normal, and the patients are asymptomatic (Morris et al. 1961; Dancis et al. 1967). They develop normally with regard to growth and intelligence; however, they are at risk for acute metabolic decompensation during stressful situations, triggered by infection or sudden increases in dietary protein. Typically, the postnatal course is uneventful, although some patients do show symptoms during the neonatal period and are diagnosed at newborn screening programs for inborn errors of metabolism (Indo et al. 1988).

MSUD is caused by dysfunction of the branched-chain $\alpha$-keto acid dehydrogenase (BCKDH) complex (Chuang and Shih 1995). BCKDH is a mitochondrial macromolecule 
multienzyme complex catalyzing the oxidative decarboxylation of branched-chain $\alpha$-keto acids derived from transamination of the branched-chain amino acids: leucine, isoleucine, and valine. The BCKDH consists of three catalytic components: a branched-chain $\alpha$-keto acid decarboxylase (E1), a dihydrolipoyl transacylase (E2), and a dihydrolipoyl dehydrogenase (E3); and two regulatory enzymes: a specific kinase and a specific phosphatase that regulate the activity of the complex through phosphorylation and dephosphorylation (Yeaman 1989; Reed and Hackert 1990). E1 is a heterotetramer of $E 1 \alpha$ and $E 1 \beta$ subunits. E1 and E2 are specific to the BCKDH, whereas E3 is shared by the family of $\alpha$-keto acid dehydrogenase complexes: $\mathrm{BCKDH}$, the pyruvate dehydrogenase complex, and the $\alpha$-ketoglutarate dehydrogenase complex. E1 catalyzes decarboxylation of the branched-chain $\alpha$-keto acids, using thiamine as a cofactor. E2 forms the structural core of the enzyme complex and catalyzes transfer of the acyl group from the $\alpha$-keto acids to coenzyme A through the lipoyl moiety covalently bound to this protein. E3 reoxidizes the lipoyl moiety of E2. E1, E3, kinase, and phosphatase are bound through noncovalent interactions.

Immunochemically, Danner et al. reported absence of the E2 subunit as a cause of MSUD (Danner et al. 1985). Subsequently, we reported that defect(s) in the E1 component are also responsible for this disease (Indo et al. 1987). Molecular defects in the BCKDH have been characterized in the classical, intermediate, and thiamine-responsive phenotypes (Chuang and Shih 1995; Indo and Matsuda 1996; Danner and McKean 1996). The classical phenotype is caused by mutation of any of the E1 $\alpha$ (Zhang et al. 1989; Matsuda et al. 1990; Fisher et al. 1991a), E1 $\beta$ (Nobukuni et al. 1991), or E2 genes (Mitsubuchi et al. 1991; Herring et al. 1992). The intermediate and thiamine-responsive phenotypes are due to mutations of $E 1 \alpha$ (Chuang et al. 1995) and E2 (Fisher et al. 1991b), respectively. More than one clinical phenotype was observed within each affected locus, since different mutations in the same subunit of BCKDH may exert dissimilar effects on the stability and function of complex proteins, leading to varying degrees of clinical manifestation (Chuang and Shih 1995). However, the molecular basis of the intermittent phenotype has remained obscure. We have now identified four novel mutations in the $E 2$ gene from three patients with intermittent MSUD, thereby demonstrating, for the first time, the molecular basis for this rare phenotype. We also characterized a rare intronic mutation, which provides a rare opportunity to consider molecular mechanisms of splicing in eukaryote transcripts.

\section{Materials and methods}

\section{Case description}

Patient KM03 was a product of consanguineous marriage between Japanese and was diagnosed at the time of newborn mass screening in Japan. Symptoms during the neona- tal period included lethargy, opisthotonos, and hypertonia, findings suggestive of the classical form of the disease. After the neonatal period, she had few attacks associated with infections and the clinical phenotype seemed to be the intermittent rather than the classical type. Mental and developmental retardation remained, probably the results of an acute metabolic episode during the neonatal period.

The second patient, KM24, was a male child of first cousin Japanese parents. Newborn mass screening for MSUD was negative. There were no clinical signs or symptoms suggestive of MSUD in the early postnatal period and developmental milestones were normal. Diagnosis of MSUD was established by analysis of his blood and urine after gastroenteritis and varicella occurred when he was 7 months old. Symptoms included elevation of body temperature, convulsion, vomiting, diarrhea, and lethargy. He was treated with intravenous glucose infusion but radical treatment such as peritoneal dialysis was not required. Thereafter, he could eat a normal diet without restriction of protein, and levels of plasma branched-chain amino acids were normal. He had several mild acute episodes, in association with infections. He survived measles with supportive therapy including intravenous glucose infusion and nasogastric feeding of a special formula free of branched-chain amino acids. At age 8 years, mental and physical development was normal. His two siblings showed no clinical signs or symptoms suggestive of MSUD. The clinical phenotype of KM24 was categorized as a typical intermittent case of MSUD.

The third patient, KM05, was a product of nonconsanguineous marriage and was diagnosed at 2 years of age, based on evidence of intermittent episodes of ketoacidosis. At 11 years of age, she could eat a normal diet without restriction of protein, and her levels of plasma branchedchain amino acids were normal. Biochemical analyses of cell lines derived from both KM03 and KM05 have been reported elsewhere (Indo et al. 1987, 1988).

\section{Enzyme analysis}

The establishment of lymphoblastoid cell lines and kinetic and immunological analyses of BCKDH proteins have been described elsewhere (Indo et al. 1987). In the case of KM24, the activity of BCKDH was determined for intact lymphoblastoid cells by quantifying ${ }^{14} \mathrm{CO}_{2}$ released from L$\left[1-{ }^{14} \mathrm{C}\right]$ leucine (Amersham, Buckinghamshire, UK).

\section{Polymerase chain reaction (PCR) analysis}

Total RNA and genomic DNAs were prepared from lymphoblastoid cells or peripheral leukocytes, using standard methods. The sequences of forward and reverse primers for BCKDH-E2 cDNA and genomic DNA are shown in Table 1. Numbering of the E2 gene was according to Lau et al. (1992a,b) . For reverse transcriptase (RT)-PCR analysis, the first-strand cDNAs were generated from $10 \mu \mathrm{g}$ of total cellular RNA with 5 pmol of random hexamer, using $200 \mathrm{U}$ of Moloney murine leukemia virus reverse transcriptase (Gibco-BRL, Gaithersburg, MD, USA) in $20 \mu \mathrm{l}$ of reaction 
Table 1 Primers used for analysis of the E2 gene

\begin{tabular}{|c|c|c|}
\hline Primer name & Primer sequences & Primer location \\
\hline \multicolumn{3}{|l|}{ Forward } \\
\hline E2-2 & 5'-GGCTGCAGTCCGTATGCTGAGAACCT-3' & $18-43$ \\
\hline E2-18 & 5'-CATTGGAGAAGGGATTAGAGAAGT-3' & $228-251$ \\
\hline E2-27 & 5'-GTATGTAAAAGAAGGAGA-3' & $267-284$ \\
\hline E2-4 & 5'-AATGGTCAAGACTATGTCTGCAGCCC-3' & $801-826$ \\
\hline E2-12 & 5'-CCTTAACGCTTCTGTGG-3' & 987-1003 \\
\hline E2-22 & 5'-TATACAGAATTCTGAAGATGGATC-3' & Intron 8 \\
\hline E2-24 & 5'-GCTGAGGTGCTTAATCCATCCAATA-3' & Intron 8 \\
\hline E2-Ex9 U & 5'-GGGTTTGATTGTCCCTAATG-3' & $1071-1090$ \\
\hline E2-Ex11 U & 5'-ACCCAGCTTTTATGCTACTAGATCTGAACT-3' & $1433-1462$ \\
\hline \multicolumn{3}{|c|}{ 200 } \\
\hline E2-1 & 5'-TGAACATGTGCTGGCACAGCTAGGGT-3' & $1514-1539$ \\
\hline $\mathrm{E} 2-5$ & 5'-GGGAGCTCAAAAAGTTCAAGAATGTC-3' & $1475-1500$ \\
\hline E2-Ex9L & 5'-ATGTTGGAAAGAGTAAATGT-3' & $1198-1217$ \\
\hline E2-7 & 5'-ACACCCTGCTCAGTATCCAT-3' & $1057-1076$ \\
\hline E2-29 & 5'-GGTTGAGCATCCCTAGTCCGTATA-3' & Intron 8 \\
\hline $\mathrm{E} 2-25$ & 5'-TAGGCAATAGGTCATTTCCCAACAT-3' & Intron 8 \\
\hline E2-3 & 5'-ATGAGGTATCTTCAGGGCTGCAGACA-3' & $815-840$ \\
\hline $\mathrm{E} 2-8$ & 5'-TTTTCCAAATAGTTGAGGAT-3' & $628-647$ \\
\hline E2-9 & 5'-ACAACATCTTCTTCTGAATC-3' & $448-467$ \\
\hline E2-28 & 5'-GATTAACAGACTTACAATACCTTTTA-3' & Junction of exon 4 and intron 4 \\
\hline
\end{tabular}

Primer location represents the location of each primer in the E2 gene according to numbering system of Lau et al. (Lau et al. 1992a) (Lau et al. 1992b).

mixture. PCR was carried out, using $2 \mu \mathrm{l}$ of the first -strand cDNA as a template and $0.5 \mu \mathrm{M}$ of each of the primers. Four sets of primers, E2-2/E2-8, E2-18/E2-3, E2-4/E2-7, and E2-12/E2-5, covering all coding exons, were used to identify mutations in the cDNA from all MSUD subjects. In addition, two sets of primers, E2-2/E2-9 and E2-18/E2-5, were used to distinguish each cDNA fragment from two different alleles in KM05. Amplification conditions were as follows: 35 cycles of denaturation at $94^{\circ} \mathrm{C}$ for $1 \mathrm{~min}$, annealing at $55^{\circ} \mathrm{C}$ for $1 \mathrm{~min}$, and extension at $72^{\circ} \mathrm{C}$ for $2 \mathrm{~min}$. Amplification of genomic DNA was performed, using the following sets of primers (the size of each product is shown in parentheses): E2-22/E2-29 (151 bp), E2-Ex11U/E2-1 (107 bp), E2-27/E2-28 (202 bp), and E2-Ex9U/E2-Ex9L (147 bp). A human genomic DNA library was screened using as a probe human E2 cDNA. A genomic DNA clone encompassing exon 8 , intron 8 , and exon 9 was isolated and characterized as described (Mitsubuchi et al. 1991). A set of primers, E224/E2-25 (265 bp), was localized to intron 8 and used for amplification of an intronic region incorporated in mRNA of KM03. The PCR reaction was done with 35 cycles, as follows: $94^{\circ} \mathrm{C}$ for $1 \mathrm{~min}, 50^{\circ}-60^{\circ} \mathrm{C}$ for $1 \mathrm{~min}, 72^{\circ} \mathrm{C}$ for $1 \mathrm{~min}$.

\section{Cloning and sequencing of PCR products}

Amplified PCR products were cloned into the T-vector (Marchuk et al. 1991).The plasmid vector, pBluescript $\mathrm{KS}(+)$ (Stratagene, La Jolla, CA, USA), was digested with $E c o \mathrm{RV}$, and treated with $T a q$ polymerase. Each clone was sequenced by a Sequenase Version 2 kit (US Biochemical, Cleveland, OH, USA) or a DNA sequencer, GENESCAN Model 373A and the Taq Dye Deoxy Terminator Cycle Sequencing Kit (PE Applied Biosystems, Foster City, CA, USA). Mutations were detected by comparing the cloned sequence with the published cDNA and genomic sequence or normal control sequence, and were confirmed if multiple clones had the same base change. To exclude a polymorphism, at least 30 normal control subjects were studied for missense mutations.

\section{Restriction digestion analysis}

For analysis of KM03, genomic DNA was amplified with a set of primers, E2-22/E2-29. PCR products were digested with AccI and electrophoresed. Conditions for PCR have already been described. For analysis of KM05, PCR products with two sets of primers, E2-27/E2-28 and E2-Ex9U/ E2-Ex9L, were digested with NlaIII and HinfI, respectively. For analysis of KM24, genomic DNA was amplified with a set of primers, E2-Ex11U/E2-1, digested with AflII. The products were incubated with each restriction enzyme and electrophoresed on 4\% NuSieve agarose gel (FMC BioProduct, San Jose, CA, USA).

\section{Exon trapping system}

An Exon Trapping System (Gibco BRL) was used to confirm the mutation in KM03 in vitro, according to the manufacturer's protocol. A 265-bp fragment of the E2 gene, including the 126-bp intronic region incorporated into mRNA of the KM03, was amplified with a primer set of E224/E2-25 from the patient and control subjects. Amplified products were cloned into the T-vector and sequenced as described. Subsequently, a XhoI-PstI fragment containing the insert from each clone was subcloned into an exon trapping vector, pSPL3 (Gibco BRL). The pSPL3 derivatives were propagated in Escherichia coli, and the DNA was 
then isolated and transfected into COS-1 cells by Lipofectin (Gibco BRL). The pSPL3 vector $(2 \mu \mathrm{g})$ and Lipofectin $(30 \mu \mathrm{l})$ were mixed and placed into $100 \mu \mathrm{l}$ of distilled water. After 15 minutes, $2 \mathrm{ml}$ of RPMI 1640 medium without fetal calf serum was added to the vector/lipid mixture. The vector/lipid/RPMI1640 medium mixture was added to COS-1 cells which were $80 \%$ confluent in the $25-\mathrm{cm}^{2}$ flask. Each flask was incubated for $6 \mathrm{~h}$ at $37^{\circ} \mathrm{C}$ in a $5 \% \mathrm{CO}_{2}$ incubator. The vector/lipid/RPMI1640 medium mixture was removed and the cells were further incubated with fresh RPMI 1640 medium containing $20 \%(\mathrm{v} / \mathrm{v})$ fetal calf serum. The following day, cells were released from the flask and mRNAs from the cells were isolated. To characterize a DNA segment trapped as an exon, mRNAs were amplified by RT-PCR, using a set of primers (SD6 and SA2) corresponding to the vector sequence.

\section{Results}

Enzyme activity of lymphoblastoid cells from MSUD patients

The decarboxylation activity of intact normal lymphoblastoid cells was $1.31 \mathrm{nmol} / \mathrm{h}$ per $\mathrm{mg}$ of cell protein. Cells from KM05 showed reduced enzyme activity: $0.12 \mathrm{nmol} / \mathrm{h}$ per mg of protein. When expressed as a percentage of the normal control level, the activity of KM05 was $9 \%$, corresponding to the value noted for disrupted cells for measurement of BCKDH directly (Indo et al. 1987). Cells from KM24 showed $20 \%$ of control activity. The enzyme activity of KM03 was similar to that of KM05, as reported earlier (Indo et al. 1988).

A single base substitution of intron 8 in the $E 2$ gene causes incorporation of an intronic region into mRNA by creating a new $5^{\prime}$ splice site and activating an upstream cryptic $3^{\prime}$ splice site

Our previous immunoblot analysis of lymphoblastoid cells from a patient, KM03, showed that the level of the E2 protein of the $\mathrm{BCKDH}$ complex was considerably decreased (Indo et al. 1988). To search for a mutation within the coding sequence, RNAs from the patient and a normal control were subjected to RT-PCR analysis with a set of primers located in exons 8 and 9, respectively. Longer and normal-sized 90-bp cDNAs were amplified; the latter was faint (Fig. 1). Nucleotide sequencing of the longer cDNA revealed that a 126-bp DNA segment was inserted into the junction between exons 8 and 9. The insertion was predicted to produce an inframe premature termination codon downstream and result in synthesis of a truncated protein with 282 amino acids, including 4 novel ones at the carboxyl terminus. These findings suggested that the RNA splicing error was responsible for the defect in this patient.

To identify the inserted DNA fragment, intron 8 of the E2 gene from a normal control was characterized. A ge-

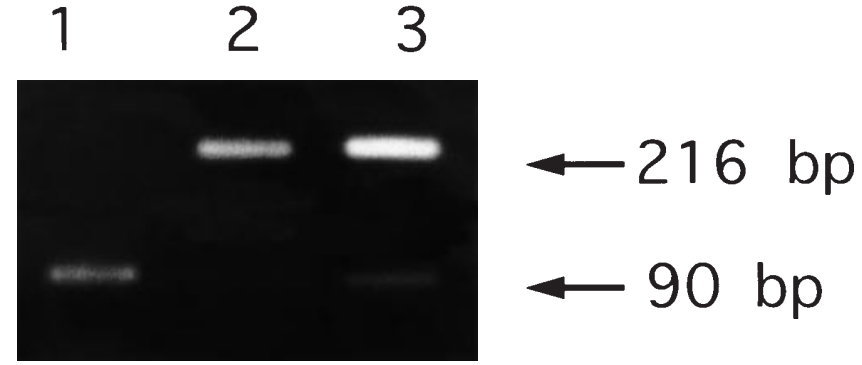

Fig. 1 Reverse transcriptase polymerase chain reaction (RT-PCR) and sequence analyses of mRNA from patient and control lymphoblastoid cells. RNA, isolated from lymphoblastoid cells derived from the patient and control, was subjected to RT-PCR analysis with a set of primers (E2-12/ E2-7; see Table 1 for sequences), for locations within exons 8 and 9 , respectively. A normal-sized 90-bp cDNA was amplified in the normal control (lane 1). In contrast, two cDNAs, one longer and one faint and normal sized, were amplified from the patient (lane 2). The intensity of these two cDNA bands was increased after changing the number of PCR cycles from 30 to 40 (lane 3). Sequence analysis showed that the longer had a 126-bp insertion between exons 8 and 9

nomic DNA clone was isolated by screening a human genomic DNA library. The clone included a region encompassing exon 8 , intron 8 , and exon 9. Intron 8 was approximately $6.8 \mathrm{~kb}$ long. Sequence analysis revealed that the 126-bp DNA insert in KM03 corresponded to a region of intron 8. The location and surrounding DNA sequences of the insert corresponding to normal control are shown in Fig. 2A and 2B, respectively.

To characterize a mutation in genomic DNA which could have led to the presumed aberrant splicing, a set of primers corresponding to intronic sequences flanking the 126-bp region involved was used. Sequence analysis revealed a single base substitution from A to G, yielding a 5' splice site adjacent to the $3^{\prime}$-end of the inserted intronic region (Fig. 3A). No sequence abnormalities were observed in the splice sites at exon 8 / intron 8 and intron 8 / exon 9 junctions. The substitution presumably led to incorporation of the 126-bp intronic region into mRNA by activating a cryptic $3^{\prime}$ splice site preceding the region.

For confirmation, restriction digestion analysis was carried out, using genomic DNAs from family members of KM03 (Fig. 3B). Genomic DNAs were amplified, digested with $A c c I$, and electrophoresed. The patient's DNA but not that from the control was digested with this enzyme, producing 128-bp and 23-bp fragments. The DNAs from the parents were partially digested, producing three bands corresponding to two digested fragments and an undigested one. Thus, the patient was homozygous and her parents were heterozygous for this mutation.

The exon trapping system is a novel approach which can be used for direct isolation of transcribed sequences from genomic DNA (Buckler et al. 1991) and we used this method to confirm the aberrant splicing in vitro. The corresponding (126-bp) and surrounding (139-bp) intronic regions of the genomic DNAs from the patient and control subjects were analyzed (Fig. 4). The 126-bp region from the patient but not from the control was recognized and recov- 
Fig. 2 Characterization of intron 8 in the E2 gene from control subject. a Schematic structure of exons 8 and 9 and intron 8. Open boxes and a shaded box indicate exons and an intronic region corresponding to the DNA inserted into the patient's mRNA, respectively. Solid lines indicate intronic regions of the approximate size shown. b Sequences of the intronic region corresponding to the DNA inserted into the patient's mRNA. Uppercase and lowercase indicate the inserted region and surrounding sequences, respectively. A base substituted in the patient is indicated by the vertical arrow. Horizontal arrows indicate the position of forward and reverse primers used to characterize this region

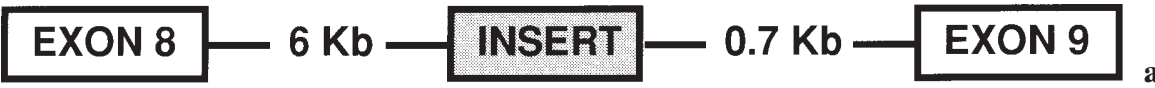

ggaatgctgaggtgcttaatccatccaataaattgaaaataaatcttta E2-24

caattctatatttgtcagTATACAGAATTCTGAAGATGGATCTTGACTC $\mathrm{E} 2-22$

AGGGTGATAAATCAGTTTTCAAGGAAATTGGCTACATCACATGACGTCAT

TTCAGCCCCCAGAAGTTTCAGATGTTGGAGCATTTCAGATTTCAGatatt 4

tggactagggatgctcaacctgtaccagttacattcccactaacgatgtt E2-29

gggaaatgacctattgcctacattcttatcaataaaaattatcatcttt $\mathrm{E} 2-25$

ttttgg

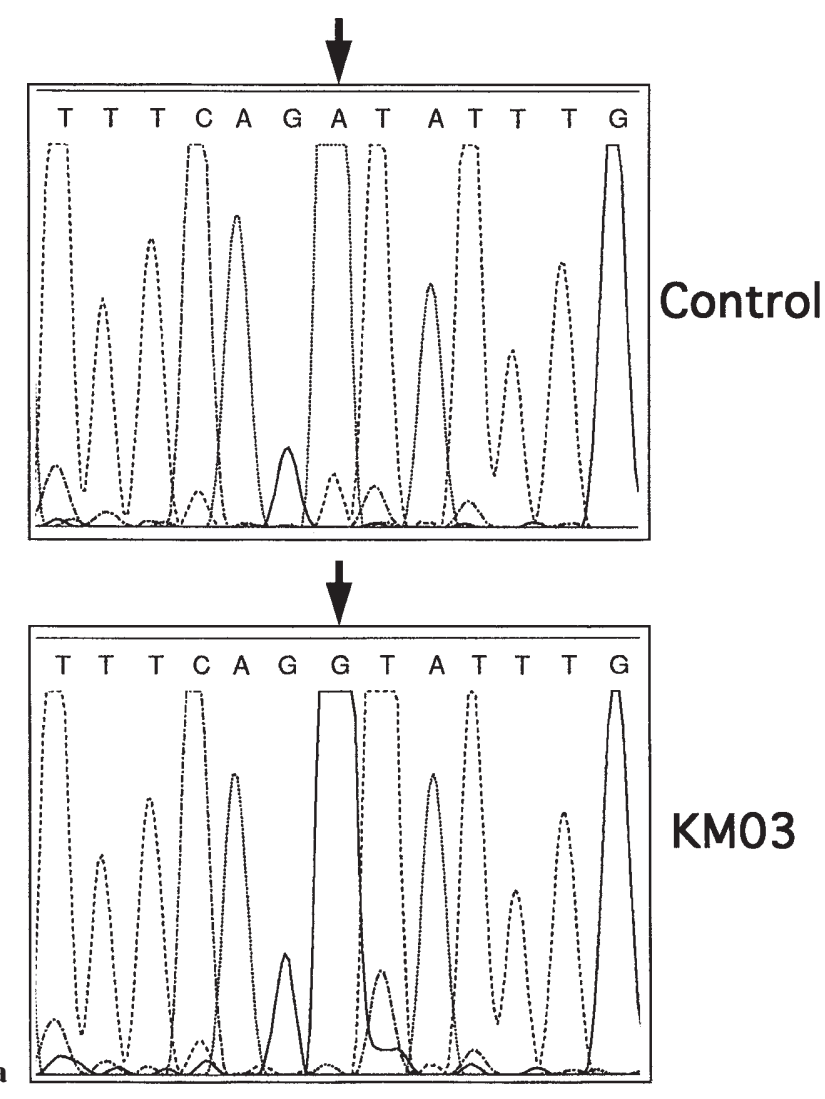

ered as an exon, thereby strongly suggesting that the single substitution was responsible for incorporation of the 126-bp region into mRNA.

A single base substitution changes the termination codon of the E2 gene to leucine and adds seven extra amino acids to the carboxyl terminus of the E2 protein

The cDNAs from KM24 were amplified, subcloned, and sequenced. We found a G-T transversion at nucleotide 1463 in exon 11 and this mutation was predicted to substi-

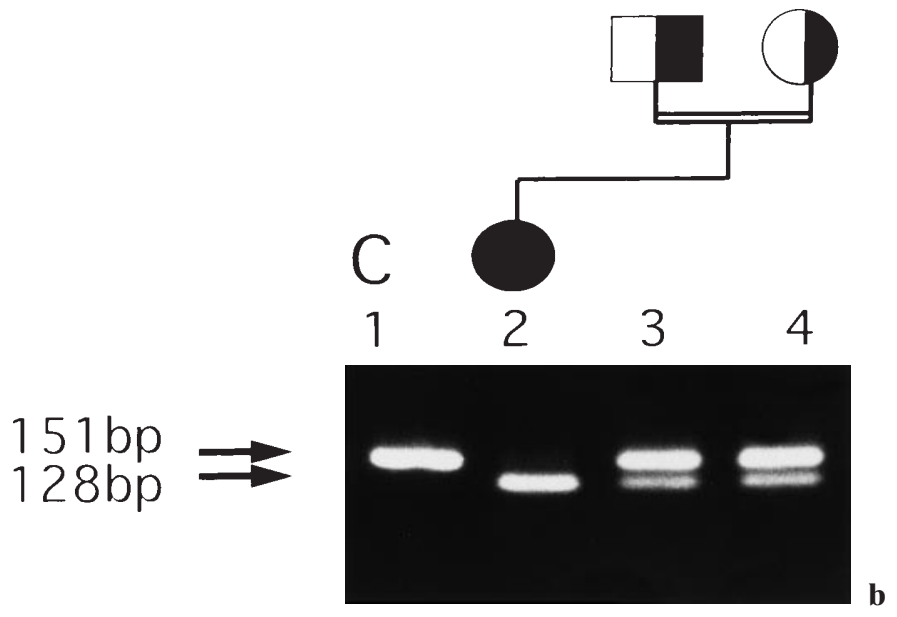

Fig. 3 a Identification of mutations in patient KM03 by DNA sequencing. To characterize genomic DNA mutation, the set of primers (E2-24/ E2-25) we used amplified the 126-bp intronic and surrounding sequences involved. These primers amplified the 265-bp region from genomic DNAs. Sequence analysis of the patient revealed a substitution from A to G (arrows), creating a novel 5' splice site adjacent to the $3^{\prime}$-end of the inserted intronic region. b Restriction digestion analysis of KM03 and her parents. Genomic DNAs were amplified with a set of primers (E2-22/E2-29), and products of $151 \mathrm{bp}$ were digested with $A c c \mathrm{I}$ and electrophoresed. There is no AccI site in the normal control sequence (lane 1). The single base substitution of the patient creates this site, providing fragments of 128 and $23 \mathrm{bp}$ (lane 2). The 23-bp fragment is not shown. Analysis of the PCR product for each individual is shown below the corresponding symbols in the pedigree

tute a leucine for the termination codon (X422L) and add seven amino acids at the carboxyl terminus of the E2 protein (Fig. 5A). To confirm this mutation, we amplified genomic DNA, and PCR products of $107 \mathrm{bp}$ were digested with $A f l$ II. There was no $A f l$ II site in the normal control sequence. The G-T transversion creates an $A f l \mathrm{II}$ site, producing cleavage fragments of $78 \mathrm{bp}$ and $29 \mathrm{bp}$. Restriction digestion analysis showed that the patient was homozygous and the consanguineous parent heterozygous for this mutation (Fig. 5B). Two siblings of the patient were also heterozygous for this mutation. 
Fig. 4 Analysis of the 126-bp intronic region from patient KM03 by the exon trap system. a Schematic structure of the construct prepared in a exon trap vector, pSPL3. The open boxes and a shaded box are vector-derived exons and the 126-bp intronic region incorporated into the mRNA of KM03, respectively. Thick and thin lines are surrounding intronic regions and vector sequences, respectively. Two restriction enzymes, XhoI and PstI, were used for subcloning. $S D v$ and $S A v$ indicate vector splice donor and vector splice acceptor sites, respectively. The corresponding regions of the genomic DNAs from the patient and control subjects were subcloned into the pSPL3. An asterisk shows an A to $\mathrm{G}$ substitution observed in KM03. b When a genomic sequence was recognized as an exon, the sequence was incorporated into the mRNA by splicing of primary transcripts. A DNA segment trapped as an exon was amplified by RTPCR, using a set of primers (SD6 and SA2) corresponding to the vector sequence. c The pSPL3 derivatives were transfected into COS-1 cells. After incubation, mRNAs were isolated from the cells and analyzed by RT-PCR. In the control subject, the vector splice donor and acceptor sequences were joined without an insert (upper section). In contrast, the 126-bp region was recognized and recovered as an exon between the vector splice donor and acceptor sequences in KM03 (lower section)
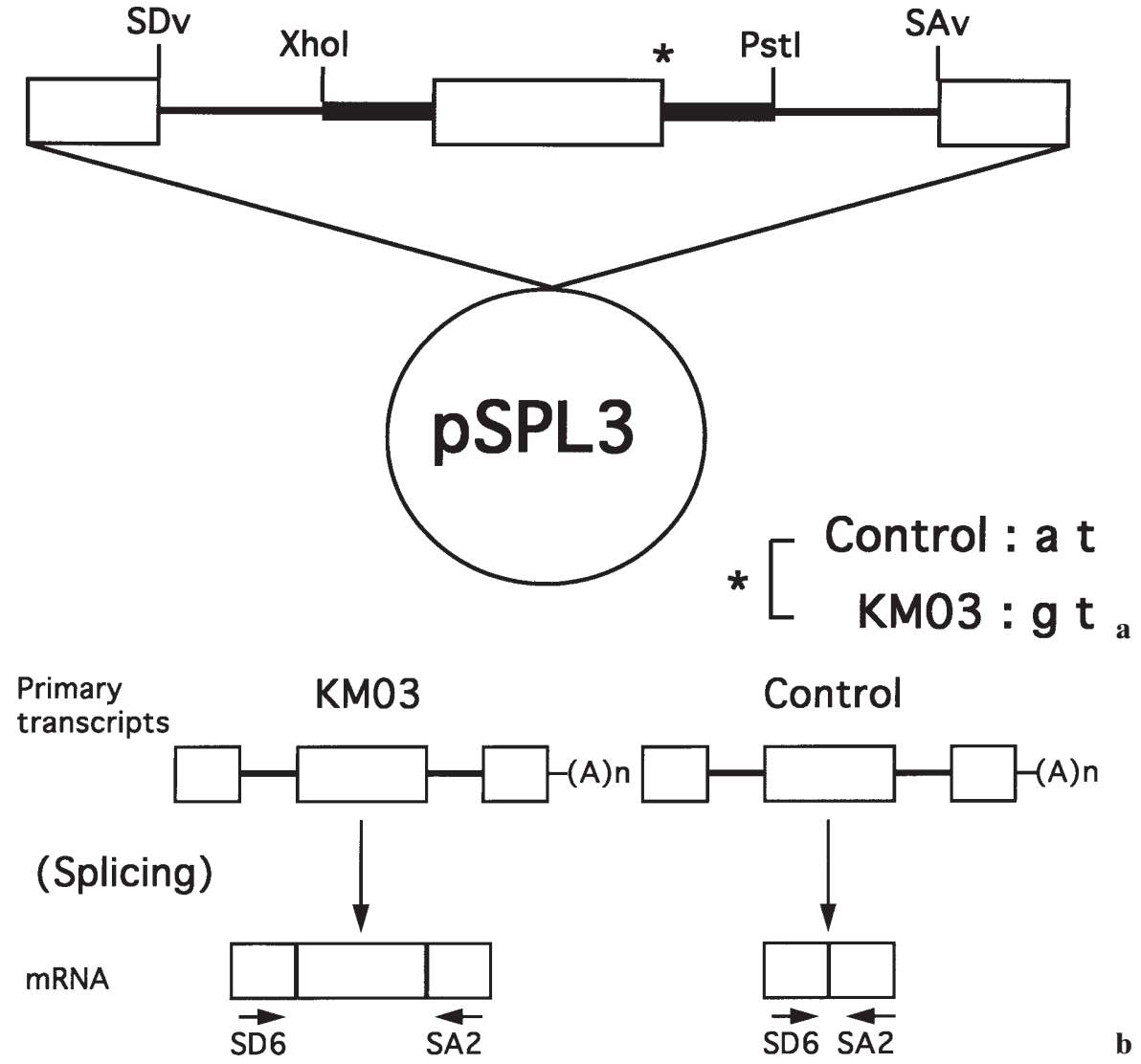

splice donor sequences $\longrightarrow$ splice acceptor sequences

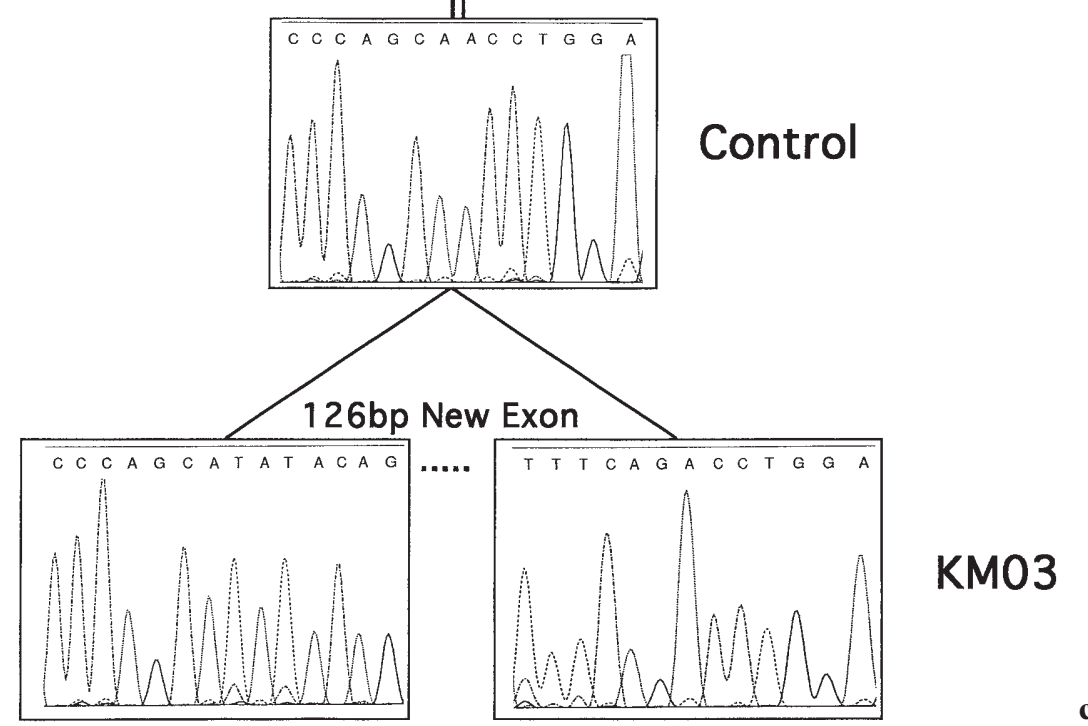

Two missense mutations of the E2 gene

Kinetic and immunological analyses of BCKDH proteins of KM05 were described elsewhere (Indo et al. 1987); the level of the E2 protein was moderately reduced in this patient. RT-PCR analyses were next performed to search for mutation(s). We found a $\mathrm{C}-\mathrm{G}$ transversion at nucleotide 309 in exon 4 and a G-A transition at nucleotide 1165 in exon 9, which cause an Ile-to-Met substitution at amino acid
37 (I37M) and a Gly-to-Ser substitution at amino acid 323 (G323S), respectively (Fig. 6A,B). Each allele was on a separate chromosome, as determined by sequencing the subcloned PCR products.

To confirm these mutations, we amplified genomic DNAs corresponding to these mutations with two sets of primers. PCR products with I37M and G323S mutations were digested with NlaIII and HinfI, respectively (Fig. 6C,D). There is no NlaIII site in the normal control 

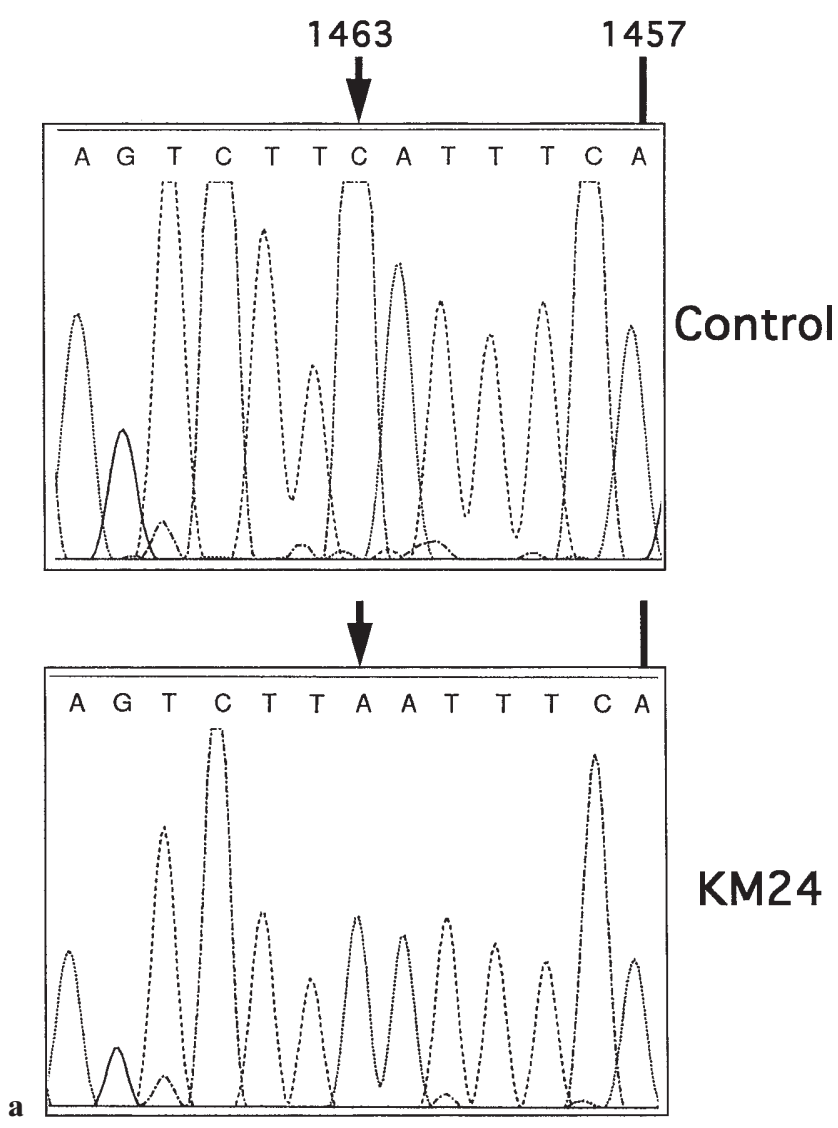

sequence. However, the $\mathrm{C}-\mathrm{G}$ transversion creates a NlaIII site, producing cleavage fragments of $159 \mathrm{bp}$ and $43 \mathrm{bp}$. As three fragments were present, the patient was likely to be heterozygous for this mutation (Fig. 6C). There was no HinfI site in the normal control sequence. The G-A transition mutation creates an HinfI site, producing cleavage fragments of $93 \mathrm{bp}$ and $54 \mathrm{bp}$. As three fragments were present, the patient was heterozygous for this mutation (Fig. 6D). Restriction digestion analyses showed that the patient was compound heterozygous for these two mutations. DNA samples from both parents were not available for restriction digestion analysis. The genomic DNAs from thirty control persons were subcloned and sequenced, and I37M mutations were never evident. The genomic DNAs were amplified from controls and digested with HinfI; there were no G323S mutations in these thirty controls.

\section{Discussion}

We identified four novel mutations in the E2 gene of the BCKDH complex from three patients with intermittent phenotype MSUD. To the best of our knowledge, this is the first molecular characterization of this unique phenotype. The E2 proteins of the BCKDH complex form the structural core of the $\mathrm{BCKDH}$, to which $\mathrm{E} 1, \mathrm{E} 3$, the kinase, and the phosphatase are attached through noncovalent interactions (Yeaman 1989; Reed and Hackert 1990). The E2 sub-

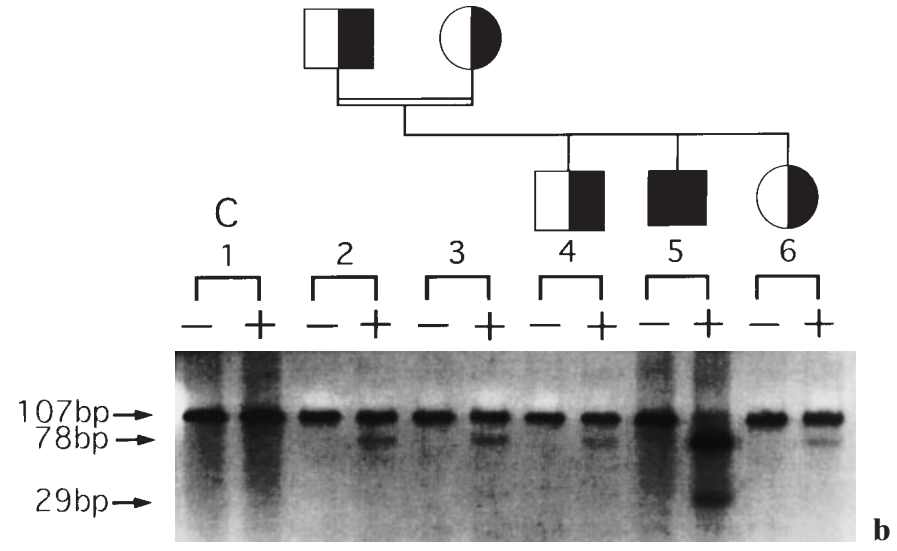

Fig. 5 Sequence and restriction digestion analysis of patient KM24. a Sequence of the region around the termination codon amplified from genomic DNA. Complementary sequences of control and KM24 are shown. A single base substitution at nucleotide 1463 of KM24 changes the termination codon (TGA) of the E2 gene to leucine (TTA) and adds seven extra amino acids to the carboxyl terminus of the E2 protein. b Restriction digestion analysis of the genomic region around the mutation in KM24 and his family members. PCR products of $107 \mathrm{bp}$ were digested with $A f I I$ and electrophoresed. The symbols (+) and $(-)$ denote presence and absence of the enzyme for incubation, respectively. There is no $A f l \mathrm{II}$ site in the normal control sequence (lane 1). The G-T transversion mutation at nucleotide 1463 creates a $A f I I$ site, producing 78-bp and 29-bp fragments. The PCR products from KM24 (lane 5) were completely digested, then 78-bp and 29-bp fragments were observed. The PCR products from the other members of the family were revealed to be heterozygous for this mutation. Analysis of the PCR product for each individual is shown below the corresponding symbols in the pedigree

unit has three domain structures: an N-terminal lipoylbearing domain, a peripheral E1/E3 subunit-binding domain, and an inner core (or C-terminal catalytic) domain. These independently folded domains are linked together via flexible hinge regions rich in alanine, proline, and negatively charged amino acid residues (Russell and Guest 1991).

We detected two novel homozygous mutations which affect the structure of the inner core domain of the E2 subunit. This domain has a transacylase active site and a region that confers the 24-mer assembly (Griffin et al. 1988; Griffin and Chuang 1990; Meng and Chuang 1994). The specific region of the inner E2 core domain indicates a strong species conservation and is highly homologous to the region of the E2 subunit of pyruvate dehydrogenase, and that of $\alpha$-ketoglutarate dehydrogenase (Russell and Guest 1991). The E2 inner core of $\alpha$-keto acid dehydrogenase complexes is assembled from trimers (Mattevi et al. 1992). According to this model, the monomers assemble into an intermediate species, active trimers, whereby constitutive polypeptides assume their final conformation. Active trimers interlock through carboxy-terminal hydrophobic knobs to produce the native 24-mer structure. We speculate that deletion of the E2 inner core or addition of extra amino acids to the carboxyl terminus might hinder the assembly of monomers into an active trimer or production of the native 24-mer structure. Thus, both mutations probably affect structure and function of the E2 protein and reduce BCKDH activity. 

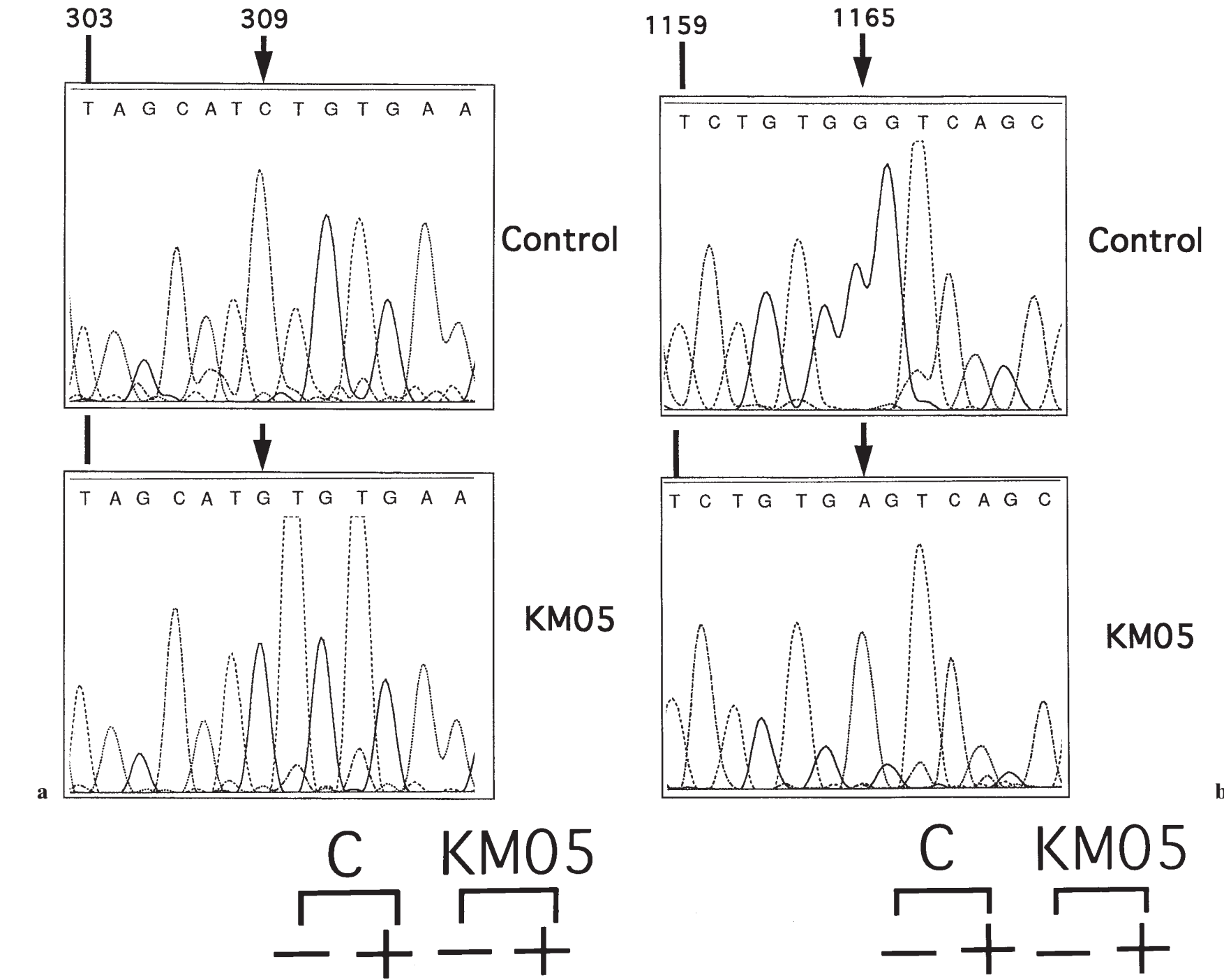
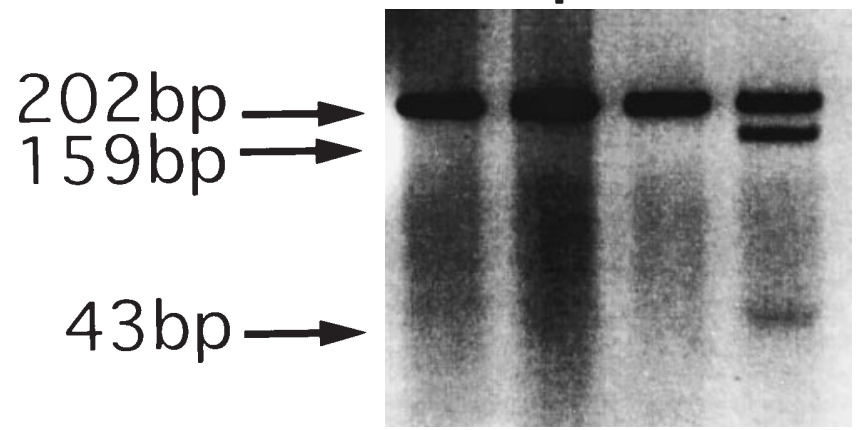

Fig. 6 Detection of two mutations in patient KM05. a Sequence analysis of the cDNA amplified with a set of primers (E2-18/E2-5) from $\mathrm{KM} 05$. A C $-\mathrm{G}$ transversion (arrows) at nucleotide 309 located in exon 4 changes codon ATC to ATG and causes an Ile-to-Met substitution at amino acid 37 (I37M). b Sequence analysis of the cDNA amplified with a set of primers (E2-18/E2-5) from KM05. A G-A transition (arrows) at nucleotide 1165 in exon 9 changes codon GGT to AGT and causes a Gly-to-Ser substitution at amino acid 323 (G323S). c Restriction digestion analysis of the genomic region around the $\mathrm{C}-\mathrm{G}$ mutation at nucleotide 309 in KM05. PCR products of $202 \mathrm{bp}$ amplified with a set of primers (E2-27/E2-28) were digested with NlaIII and electrophoresed on a $4 \%$ agarose gel. The symbols $(+)$ and $(-)$ denote presence and absence of the enzyme for incubation, respectively. There is no NlaIII

\section{$147 \mathrm{bp} \longrightarrow$
$93 \mathrm{bp} \longrightarrow$
$54 \mathrm{bp} \longrightarrow$}

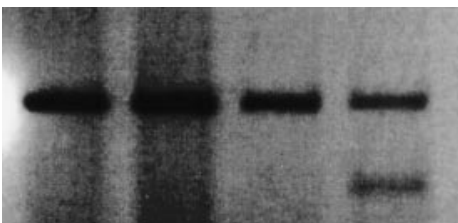

site in the normal control sequence (lanes 1,2). The $\mathrm{C}-\mathrm{G}$ mutation at nucleotide 309 in KM05 creates an NlaIII site and the PCR product from the patient was digested, providing fragments of $159 \mathrm{bp}$ and $43 \mathrm{bp}$ (lanes 3,4). The presence of a 202-bp band in KM05 with digestion indicates that the patient is a compound heterozygote. d Restriction digestion analysis in the genomic region around the $\mathrm{G}-\mathrm{A}$ transition at nucleotide 1165 in exon 9. PCR products of $147 \mathrm{bp}$ amplified with a set of primers (E2-Ex9U/E2-Ex9L) were digested with HinfI. There is no HinfI site in the normal control sequence (lanes 1,2). The G-A mutation creates a HinfI site, producing 93-bp and 54-bp fragments (lanes $3,4)$. The presence of a 147-bp band in KM05 with digestion indicates that the patient is a compound heterozygote 
The third patient was a compound heterozygote for missense mutations in the E2 gene. These two mutations, I37M and G323S, were located in the lipoyl-bearing and the inner core domains, respectively. The domain sequences are sufficiently well-conserved in evolution and analogous segments can be clearly identified in acyltransferase subunits (E2) of all $\alpha$-keto acid dehydrogenase complexes (Russell and Guest 1991). The lipoyl domain(s) of the acyltransferases perform essential roles in the reaction mechanism of the $\alpha$-keto acid dehydrogenase complexes, carrying substrate and reducing equivalents between active sites of the complexes. One mutation, I37M, changed an amino acid residue which was either Ile, Leu, or Val and located between two perfectly conserved residues in all E2 sequences: a glycine (Gly-28) and a lipoyl-lysine (Lys-44). The other mutation, G323S, was located in the inner core domain and changed an amino acid residue conserved among 9 of the 13 acyltransferase sequences. The BCKDH enzyme activity was about one-tenth that of the normal control and the level of E2 protein was moderately reduced in this patient. It is likely that either or both of the two putative mutant proteins have reduced stability. Studies of the expression of each allele from this patient are ongoing.

The residual BCKDH activities of these patients with intermittent phenotype were significantly higher than those of the patient with classical phenotype (Indo et al. 1988; Dancis et al. 1972). This might suggest that defect(s) of the E2 subunit confer more residual BCKDH activity than do those of the E1 $\alpha$ or E1 $\beta$ subunit. A plausible explanation is that the stability of the E1 or E2 components is probably maintained independently by an intrinsic quality-control system in the mitochondria, and that the E1 protein might be able to function by itself, catalyzing decarboxylation, under certain conditions. Alternatively, the possibility of cross-reaction between the components (subunits) of the $\alpha$ keto acid dehydrogenase complexes, including the pyruvate dehydrogenase, would need to be considered. The overall reaction of the $\mathrm{BCKDH}$ complex is similar to that of the pyruvate dehydrogenase complex and these two complexes share the same E3 component. The enzyme components of these two complexes do not interact to any significant extent (Cook et al. 1985). However, the low but significant BCKDH activity in patients with the E2 mutation suggests that interaction of E1 and E2 components of other $\alpha$-keto acid dehydrogenase complexes might occur in the presence of a pathology, the result being the reconstitution of decarboxylation.

This notion is further supported by a study reporting that the originally described MSUD patient (WG-34) with the thiamine responsive phenotype is a compound heterozygote of the mutated E2 gene (Fisher et al. 1991b; Scriver et al. 1971). This patient showed a marked and immediate reduction in plasma branched-chain amino acids when her protein-restricted diet was supplemented with a pharmacological dose of thiamine. A follow-up report on this patient suggested that several episodes of metabolic decompensation occurred while she was on thiamine supplementation (Scriver et al. 1985). This case might be categorized as intermittent phenotype MSUD, although the mechanism in- volved in the clinical response to thiamine remains to be determined.

We reported the case of a patient with classical MSUD and a deletion of the inner E2 core domain (Mitsubuchi et al. 1991). The enzyme activity in this patient was less than that of the intermittent case but higher than that of classical case. An inframe 78-base deletion in mRNA was caused by a skipping of exon 8 due to a single base deletion at the $5^{\prime}$-splice donor site. The deletion produced a truncated $\mathrm{E} 2$ protein lacking 26 amino acids (253-278), which was weakly visible on the immunoblot. Different clinical phenotypes were observed in two patients, both with the truncated E2 protein. This might be explained in part by differences in residual enzyme activity. A trace amount of the normal-sized transcript would probably confer some activity in the present case.

Splicing converts primary transcripts of the eukaryotic cell into mRNAs that are then exported to the cytoplasm (Sharp 1994). The general mechanism which has been proposed suggests the formation of a spliceosome complex containing small nuclear ribonucleoproteins as well as newly transcribed RNA - which is involved in recognition and splicing at appropriate $5^{\prime}$ and $3^{\prime}$ sites. Mechanisms by which all introns are excised from genes containing multiple introns have been proposed but not established. A 5'-to-3' directional scanning model was proposed, in which the spliceosomal complex moves linearly along the primary transcripts, selecting $5^{\prime}$ and $3^{\prime}$ splice sites as it proceeds (Lang and Spritz 1983). Such a model cannot explain the mutation described in our patient, in whom creation of the novel $5^{\prime}$ splice site causes recognition of an upstream intronic region as an exon. Further, this scanning model cannot explain abnormal splicing in some patients with inherited disorders, in whom mutations in $5^{\prime}$ splice sites cause skipping of the previous exon (Krawczak et al. 1992).

Alternatively, an exon definition model in which the spliceosome recognizes a $3^{\prime}$ splice site and then scans downstream for an acceptable $5^{\prime}$ splice site, thereby defining an exon (Robberson et al. 1990; Niwa et al. 1992) has been proposed. In this model, spliceosome assembly in primary transcripts can be initiated through interaction between the $3^{\prime}$ splice site and specific sequence elements on the downstream exon (or a $5^{\prime}$ splice site). Subsequently, pairing of the intronic $5^{\prime}$ and $3^{\prime}$ splice sites probably occurs in the distinct steps (Chiara and Reed 1995). Analysis of the unique splice mutation observed in our patient favors the exon definition model in eukaryotic splicing.

Acknowledgments We thank M. Ohara for assistance with the manuscript, and family members of the patients for their cooperation. This work was funded in part by a Grant-in-Aid for Scientific Research (C) from The Ministry of Education, Science, Sports, and Culture of Japan, and The Research Grant (8A-7) for Nervous and Mental Disorders and a Grant for Pediatric Research from The Ministry of Health and Welfare of Japan.

\section{References}

Buckler AJ, Chang DD, Graw SL, Brook JD, Haber DA, Sharp PA, Housman DE (1991) Exon amplification: a strategy to isolate mam- 
malian genes based on RNA splicing. Proc Natl Acad Sci USA 88: 4005-4009

Chiara MD, Reed R (1995) A two-step mechanism for 5' and 3' splicesite pairing. Nature 375 : $510-513$

Chuang DT, Shih VE (1995) Disorders of branched chain amino acid and keto acid metabolism. In: Scriver CR, Beaudet AL, Sly WS, Valle D (eds) The metabolic and molecular bases of inherited disease, 7th edn. McGraw-Hill, New York, pp 1239-1277

Chuang JL, Davie JR, Chinsky JM, Wynn RM, Cox RP, Chuang DT (1995) Molecular and biochemical basis of intermediate maple syrup urine disease. Occurrence of homozygous G245R and F364C mutations at the $E 1 \alpha$ locus of Hispanic-Mexican patients. J Clin Invest 95: 954-963

Cook KG, Bradford AP, Yeaman SJ (1985) Resolution and reconstitution of bovine kidney branched-chain 2-oxo acid dehydrogenase complex. Biochem J 225: 731-735

Dancis J, Hutzler J, Rokkones T (1967) Intermittent branched-chain ketonuria - variant of maple-syrup-urine disease. N Engl J Med 276: 84-89

Dancis J, Hutzler J, Snyderman SE, Cox RP (1972) Enzyme activity in classical and variant forms of maple syrup urine disease. J Pediatr 81 : 312-320

Danner DJ, McKean MC (1996) Multigenic basis for maple syrup urine disease with emphasis on mutations in branched chain dihydrolipoyl acyltransferase. In: Patel MS, Roche TE, Harris RA (eds) Alphaketo acid dehydrogenase complexes. Birkhäuser, Basel, pp 271-293

Danner DJ, Armstrong N, Heffelfinger SC, Sewell ET, Priest JH, Elsas LJ (1985) Absence of branched chain acyl-transferase as a cause of maple syrup urine disease. J Clin Invest 75: 858-860

Fisher CR, Fisher CW, Chuang DT, Cox RP (1991a) Occurrence of a Tyr393 $\rightarrow$ Asn $(Y 393 N)$ mutation in the E1 $\alpha$ gene of the branchedchain $\alpha$-keto acid dehydrogenase complex in maple syrup urine disease patients from a Mennonite population. Am J Hum Genet 49: 429-434

Fisher CW, Lau KS, Fisher CR, Wynn RM, Cox RP, Chuang DT (1991b) A 17-bp insertion and a Phe215-Cys missense mutation in the dihydrolipoyl transacylase (E2) mRNA from a thiamineresponsive maple syrup urine disease patient WG-34. Biochem Biophys Res Commun 174: 804-809

Griffin TA, Chuang DT (1990) Genetic reconstruction and characterization of the recombinant transacylase (E2b) component of bovine branched-chain $\alpha$-keto acid dehydrogenase complex. Implication of histidine 391 as an active site residue. J Biol Chem 265: 13174-13180

Griffin TA, Lau KS, Chuang DT (1988) Characterization and conservation of the inner E2 core domain structure of branched-chain $\alpha$ keto acid dehydrogenase complex from bovine liver. Construction of a cDNA encoding the entire transacylase (E2b) precursor. J Biol Chem 263: 14008-14014

Herring WJ, McKean M, Dracopoli N, Danner DJ (1992) Branched chain acyltransferase absence due to an Alu-based genomic deletion allele and an exon skipping allele in a compound heterozygote proband expressing maple syrup urine disease. Biochim Biophys Acta 1138: 236-242

Indo Y, Matsuda I (1996) Molecular defects of the branched-chain $\alpha$ keto acid dehydrogenase complex: maple syrup urine disease due to mutations of the E1 $\alpha$ or E1 $\beta$ subunit gene. In: Patel MS, Roche TE, Harris RA (eds) Alpha-keto acid dehydrogenase complexes. Birkhäuser, Basel, pp 227-247

Indo Y, Kitano A, Endo F, Akaboshi I, Matsuda I (1987) Altered kinetic properties of the branched-chain $\alpha$-keto acid dehydrogenase complex due to mutation of the $\beta$-subunit of the branched-chain $\alpha$ keto acid decarboxylase (E1) component in lymphoblastoid cells derived from patients with maple syrup urine disease. J Clin Invest 80: $63-70$

Indo Y, Akaboshi I, Nobukuni Y, Endo F, Matsuda I (1988) Maple syrup urine disease: a possible biochemical basis for the clinical heterogeneity. Hum Genet 80: 6-10
Krawczak M, Reiss J, Cooper DN (1992) The mutational spectrum of single base-pair substitutions in mRNA splice junctions of human genes: causes and consequences. Hum Genet 90: 41-54

Lang KM, Spritz RA (1983) RNA splice site selection: evidence for a $5^{\prime} \rightarrow 3^{\prime}$ scanning model. Science 220: 1351-1355

Lau KS, Chuang JL, Herring WJ, Danner DJ, Cox RP, Chuang DT (1992a) The complete cDNA sequence for dihydrolipoyl transacylase (E2) of human branched-chain $\alpha$-keto acid dehydrogenase complex. Biochim Biophys Acta 1132: 319-321

Lau KS, Herring WJ, Chuang JL, McKean M, Danner DJ, Cox RP, Chuang DT (1992b) Structure of the gene encoding dihydrolipoyl transacylase (E2) component of human branched chain $\alpha$-keto acid dehydrogenase complex and characterization of an E2 pseudogene. J Biol Chem 267: 24090-24096

Marchuk D, Drumm M, Saulino A, Collins FS (1991) Construction of T-vectors, a rapid and general system for direct cloning of unmodified PCR products. Nucleic Acids Res 19: 1154

Matsuda I, Nobukuni Y, Mitsubuchi H, Indo Y, Endo F, Asaka J, Harada A (1990) A T-to-A substitution in the E1 $\alpha$ subunit gene of the branched-chain $\alpha$-ketoacid dehydrogenase complex in two cell lines derived from Mennonite maple syrup urine disease patients. Biochem Biophys Res Commun 172: 646-651

Mattevi A, Obmolova G, Schulze E, Kalk KH, Westphal AH, de KA, Hol WG (1992) Atomic structure of the cubic core of the pyruvate dehydrogenase multienzyme complex. Science 255: 1544-1550

Meng M, Chuang DT (1994) Site-directed mutagenesis and functional analysis of the active-site residues of the E2 component of bovine branched-chain $\alpha$-keto acid dehydrogenase complex. Biochemistry 33: $12879-12885$

Mitsubuchi H, Nobukuni Y, Akaboshi I, Indo Y, Endo F, Matsuda I (1991) Maple syrup urine disease caused by a partial deletion in the inner $\mathrm{E} 2$ core domain of the branched chain $\alpha$-keto acid dehydrogenase complex due to aberrant splicing. A single base deletion at a $5^{\prime}$ splice donor site of an intron of the $E 2$ gene disrupts the consensus sequence in this region. J Clin Invest 87: 1207-1211

Morris MD, Lewis BD, Doolan PD, Harper HA (1961) Clinical and biochemical observations on an apparently nonfatal variant of branched-chain ketoaciduria (maple syrup urine disease). Pediatrics 28: 918-923

Niwa M, MacDonald CC, Berget SM (1992) Are vertebrate exons scanned during splice-site selection? Nature 360: 277-280

Nobukuni Y, Mitsubuchi H, Akaboshi I, Indo Y, Endo F, Yoshioka A, Matsuda I (1991) Maple syrup urine disease. Complete defect of the E1 $\beta$ subunit of the branched chain $\alpha$-ketoacid dehydrogenase complex due to a deletion of an 11-bp repeat sequence which encodes a mitochondrial targeting leader peptide in a family with the disease. J Clin Invest 87: 1862-1866

Reed LJ, Hackert ML (1990) Structure-function relationships in dihydrolipoamide acyltransferases. J Biol Chem 265: 8971-8974

Robberson BL, Cote GJ, Berget SM (1990) Exon definition may facilitate splice site selection in RNAs with multiple exons. Mol Cell Biol 10: 84-94

Russell GC, Guest JR (1991) Sequence similarities within the family of dihydrolipoamide acyltransferases and discovery of a previously unidentified fungal enzyme. Biochim Biophys Acta 1076: 225232

Scriver CR, Mackenzie S, Clow CL, Delvin E (1971) Thiamineresponsive maple-syrup-urine disease. Lancet 1: 310-312

Scriver CR, Clow CL, George H (1985) So-called thiamin-responsive maple syrup urine disease: 15 -year follow-up of the original patient. J Pediatr 107: 763-765

Sharp PA (1994) Split genes and RNA splicing. Cell 77: 805-815

Yeaman SJ (1989) The 2-oxo acid dehydrogenase complexes: recent advances. Biochem J 257: 625-632

Zhang B, Edenberg HJ, Crabb DW, Harris RA (1989) Evidence for both a regulatory mutation and a structural mutation in a family with maple syrup urine disease. J Clin Invest 83: 1425-1429 